\title{
Techniques générales uttilisant le stockage d'air comprimé Perspectives d'avenir
}

\author{
par A. Charrier \\ J. Masedrello \\ Ingénicur-Conseil \\ Chef du Département Matériel \\ el R. Roudier \\ lngénicur \\ S.E.P.T.E.N., Direction de l'Equipement de l'Electricité de France
}

Le principe de l'accumulation pneumatique a été dégagé il y a une vingtaine d'années. Il consiste à stocker de l'air comprimé dans une cavité souterraine pendant les heures creuses au moyen d'un compresseur entraîné par un moteur électrique. La restitution de l'énergie s'effectue aux heures de pointe après passage de cet air dans une chambre de combustion et détente dans une turbine à gaz. En tant que procédé de transfert de l'énergie électrique, l'accumulation pneumatique peut se comparer à l'accumulation hydraulique. En France, depuis 1966 notamment, des études technicoéconomiques ont été développées, pour fixer les performances et les coûts de ce type d'installation, par la Société Générale d'Etudes et d'Equipement. Depuis cette date, des réservoirs de stockage de gaz naturel sont exploités par le gaz de France. Ces réservoirs sont en structure poreuse comme à Beynes ou creusés dans le sol à grande profondeur, en poche saline, comme à Tersannes. Les sociétés pétrolières ont construit, avec l'aide de Geostock, des cavernes rocheuses pour le stockage de pétrole brut et de gaz liquéfiés. Dans la région parisienne la création d'un réservoir de $10^{6} \mathrm{~m}^{3}$ est prévue.

Des projets de centrale aves accumulation pneumatique sont à l'étude à l'étranger. Une société allemande, la Nord West Deutsche Kraftwerke envisage une centrale d'énergie de pointe capable de délivrer une puissance maximale de $400 \mathrm{MW}$. Une étude est en cours pour le Danemark à la Société Brown Boveri. Dans ces deux installations, les réservoirs d'air seront creusés en poche saline et fonctionneront à pression variable, sans compensation hydraulique de l'air soutiré.

Depuis 1966, nous avons une meilleure connaissance des problèmes de construction des réservoirs souterrains. En ce qui concerne le matériel nous savons que la technique des turbines à gaz évolue vers les hautes températures et les grandes puissances. Il a donc paru nécessaire de refaire le bilan des installations à transfert d'énergie par accumulation d'air comprimé.

Les études d'implantation ont montré que les sites les plus favorables seraient situés dans l'ouest (Bretagne), le nord ou le Bassin parisien. En dehors des réservoirs naturels (terrains poreux ou cavernes) dont la découverte est aléatoire, les réservoirs les mieux adaptés à ces régions sont des réservoirs creusés dans le roc avec compensation hydrauli- que. L'air stocké est maintenu sous une pression presque constante par une colonne d'eau, et le volume du réservoir est utilisé au mieux, car l'eau vient remplacer l'air au fur et à mesure de son utilisation. La présente étude n'envisagera donc que des installations à réservoirs avec compensation hydraulique et donnera dans ses grandes lignes les caractéristiques du matériel utilisé.

L'évaluation économique donne des coûts relativement élevés par $\mathrm{kW}$ installé. L'évolution vers des pressions supérieures à 25 bars dans le réservoir et supérieures à 10 bars à ladmission de la turbine à gaz et surtout la mise en cuvre de l'injection d'eau dans la chambre de combustion, permettront d'obtenir des coûts beaucoup plus attractifs dans l'avenir.

Toutefois cette évaluation implique, de la part des constructeurs de turbines à gaz, l'étude et la construction d'un matériel spécialement adapté. Une appréciation correcte des besoins à venir, ainsi qu'une bonne expérience de l'exploitation des réservoirs à compensation hydraulique semble nécessaire avant que des installations industrielles ne voient le jour.

Généralités

Le stockage de l'énergie peut se faire aux heures creuses, donc pendant environ huit heures par jour de $22 \mathrm{~h}$ à $6 \mathrm{~h}$ du matin. L'installation doit permettre de fournir diverses sortes d'énergie, l'énergie de pointe deux à trois heures par jour, l'énergie de jour pendant les mois chargés.

Nous choisirons une durée de production de $4 \mathrm{~h}$ par jour. Le transfert d'énergie se fait avec un coefficient d'amplification qui résulte de l'apport d'énergie du combustible dans la chambre de la turbine à gaz. Il est commode de considérer l'inverse de ce coefficient:

$$
e 1=\mathrm{E} a / \mathrm{E} p
$$

Ea énergie dépensée pour la compression de l'air;

$\mathrm{E} p$ énergie produite par la détente des gaz;

$e 1$ dépend des conditions thermodynamiques (pression et température de gaz à l'admission). 


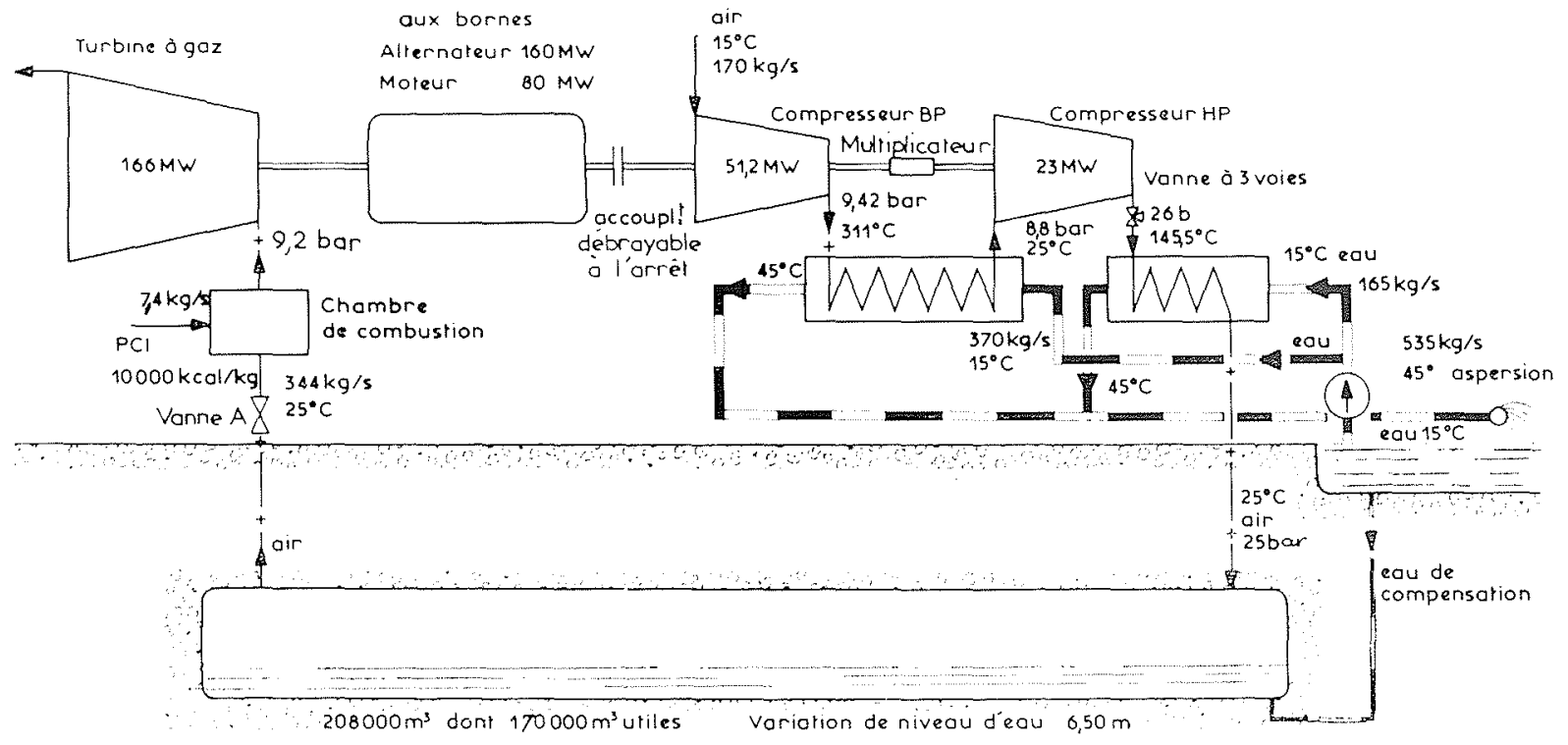

1/ Centrale de pointe avec accumulation dair comprimé.

L'installation est d'autant plus économique que e1 est plus petit.

La quantité de combustible nécessaire pour produire $1 \mathrm{kWh}$ d'énergie est caractérisée par le coefficient:

$$
e 2=860 / Q
$$

Q kilocalories apportées par le combustible, par $\mathrm{kWh}$ produit en énergie électrique;

$e 2$ comme $e 1$ dépend des conditions thermodynamiques adoptées.

La consommation spécifique en $\mathrm{kcal} / \mathrm{kWh}$ comprendra deux parties. D'une part, la quantité de chaleur Q apportée par kWh dans la chambre de combustion, d'autre part, une certaine quantité de chaleur nécessaire pour fournir l'énergie de compression, soit :

$$
\mathrm{Q}^{\prime}=e 1 \times 2200 \mathrm{kcal}
$$

Nous prendrons une consommation spécifique moyenne de $2200 \mathrm{kcal}$ par $\mathrm{kWh}$ nécessaire à la compression.

$\mathrm{La}$ consommation spécifique totale $\mathrm{Q} t$ par $\mathrm{kWh}$ produit est donc:

$$
\mathrm{Q} t=\mathrm{Q}+\mathrm{Q}^{\prime}=(860 / e 2)+2200 e 1
$$

Elle n'est qu'un des éléments du choix du niveau d'utilisation de l'usine dans l'ensemble du parc des centrales et doit être complétée par la notion de coût proportionnel du $\mathrm{kWh}$ produit. Soit $\mathrm{C} t$ ce coût, nous pouvons écrire :

$$
\mathrm{C} t=f \mathrm{Q}+e \mathrm{C}
$$

$f$ coût du combustible brûlé en turbine à gaz;

$\mathrm{C}$ coût du $\mathrm{kWh}$ fourni par la réseau (en heures creuses) avec groupes motocompresseurs.

Ce coût C varie suivant l'origine de l'énergie de compression. Il est environ 2,5 fois plus faible pour un $\mathrm{kWh}$ d'origine nucléaire que pour un $\mathrm{kWh}$ d'origine thermique.

Pour effectuer un premier projet type nous avons fixé la pression du réservoir à 25 bars absolus, pression qu'il apparaît le plus probable de réaliser dans les régions de France précitées, compte tenu de la géologie des terrains sous jacents. La turbine de détente est celle qui correspond aux plus grosses machines fabriquées actuellement par les principaux constructeurs de turbines à gaz $(350 \mathrm{~kg} / \mathrm{s}$ en débit de gaz). Comparé avec une turbine à gaz à cycle simple, ce mode de production d'énergie comporte des éléments économiques favorables. La puissance installée est trois fois plus élevée pour un matériel donné et le coût différentiel de l'énergie accumulée ne coûte que les frais de combustible pour la produire et les pertes en ligne. Il valorise les centrales de base (centrales nucléaires notamment) par le transfert de l'énergie électrique qu'il effectue. Il comporte aussi des éléments économiques défavorables qui sont le prix du réservoir avec son équipement et le prix du transfert d'énergie en heures pleines.

\section{Avant-projet type d'installation}

\section{Description du matériel.}

Le schéma de l'installation mécanique est indiqué sur la figure 1 .

La ligne d'arbre comprend la turbine à gaz, l'alternateur, un accouplement débrayable à l'arrêt, le compresseur basse pression, un multiplicateur de vitesse, le compresseur haute pression.

Entre le réservoir souterrain et la turbine à gaz, se trouvent: une vanne de laminage qui ramène la pression de 25 à 10 bars, éventuellement une vanne d'arrêt et la chambre de combustion.

Une pompe de circulation envoie un débit d'eau de $0,535 \mathrm{~m}^{3} / \mathrm{s}$ dans les réfrigérants d'air comprimé; à la sortie du compresseur HP une vanne à trois voies permet une mise à l'air libre. 


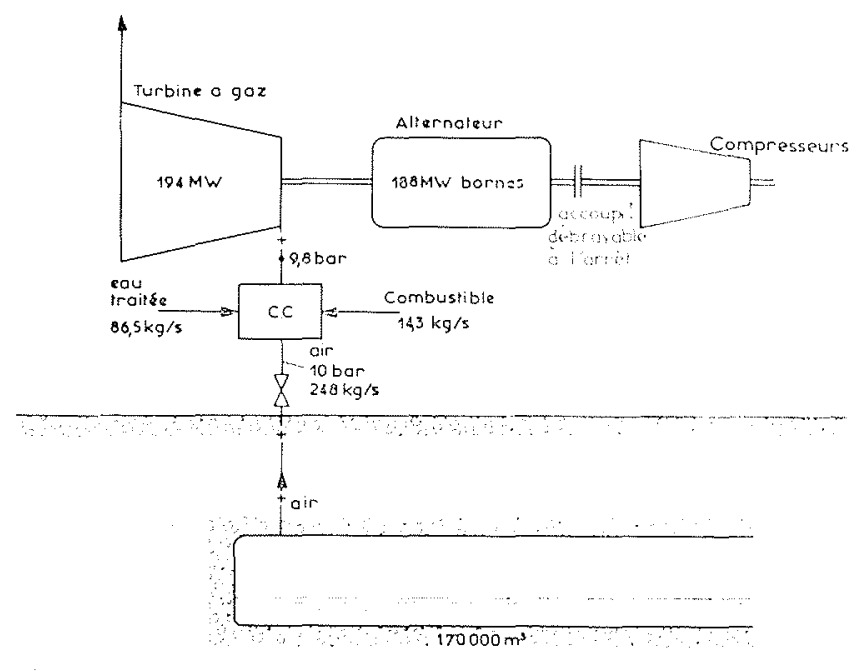

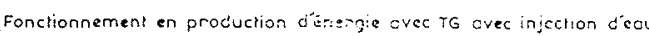

2/ Centrale de pointe avec accumulation d'air comprimé.

\section{Fonclionnement ef performance.}

\section{Heures Creuses :}

La turbine à gaz sert au démarrage de l'alternateur accouplé aux compresseurs, Lorsque la ligne d'arbre est à 3000 tours, l'alternateur, fonctionnant en moteur, entraîne les compresseurs, tandis que la vanne A est fermée. La turbine tourne en ventilateur.

Un premier remplissage partiel du réservoir est nécessaire pour assurer le premier démarrage, il se fera avec un compresseur auxiliaire à faible débit.

Suivant le schéma (fig. 1), les compresseurs fonctionnant $8 \mathrm{~h}$ par jour délivrent un débit d'air de $170 \mathrm{~kg} / \mathrm{s}$ à $25^{\circ} \mathrm{C}$ sous 26 bars. La puissance d'entraînement nécessaire est d'environ $80 \mathrm{MW}\left({ }^{*}\right)$.

\section{Heures pleines:}

La turbine à gaz entraîne l'alternateur pour fournir une puissance aux bornes de $160 \mathrm{MW}$. Son fonctionnement prévu est de quatre heures par jour $(*)$. Un accouplement débrayable à l'arrêt, permet de stopper les compresseurs. La vanne A est ouverte. La vanne à trois voies $\mathrm{T}$ est fermée côté réservoir d'air comprimé

Compte tenu de l'énergie utilisable de l'air comprimé, la quantité de chaleur $\mathrm{Q}$ apportée dans la chambre de combustion sera de $1700 \mathrm{kcal} \mathrm{PCS} / \mathrm{kWh}$; nous aurons donc:

$$
e 2=0,505
$$

el étant égal à 1 , aux pertes près dans les lignes et le transformateur, la consommation spécifique équivalente totale serait $\mathrm{Q}+\mathrm{Q}^{\prime}=3900 \mathrm{kcal} \mathrm{PCS} / \mathrm{kWh}$ produit pour une

(*) La masse spécifique de l'air, à $25^{\circ} \mathrm{C}$ sous 25 bars, qui se trouve dans le réservoir est de $29,2 \mathrm{~kg} / \mathrm{m}^{3}$. Pour une foumiture d'énergie pendant quatre heures par jour avec un débit d'air de $344 \mathrm{~kg} / \mathrm{s}$ il faut donc une capacite utile de $170000 \mathrm{~m}^{3}$ environ.

Les compresseurs fonctionnant pendant huit heures par jour au minimum, leur débit d'air est moitié de celui de la turbine. production d'énergie de $3,8 \mathrm{kWh} / \mathrm{m}^{3}$ utile de réservoir. Ces performances assez peu élevées sont dues aux rendements des machines et au laminage de l'air entre le réservoir souterrain et la chambre de combustion. Elles correspondent au point $\mathrm{A}$ de la figure 3. Il existe plusieurs possibilités pour les améliorer.

\section{Possibilité d'amélioration des performances}

\section{Injection d'eau.}

Il est possible de réaliser une chambre de combustion dans laquelle l'air est brûlé avec un excès d'air de $10 \%$. Les gaz sont alors refroidis par une injection d'eau avant admission dans la turbine à gaz. Plusieurs sociétés seraient en mesure de développer une telle chambre. Les performances obtenues avec une turbine dont le débit de gaz est de $350 \mathrm{~kg} / \mathrm{s}$ sont reproduites sur le schéma de la figure 2 . La pression d'admission turbine a été légèrement augmentée, 9,8 au lieu de 9,2 bars.

Nous avons :

— débit d'air nécessaire : $248 \mathrm{~kg} / \mathrm{s}$ au lieu de 344 ;

- puissance alternateur: $188 \mathrm{MW}$ au lieu de 160 .

On obtient donc avec le même réservoir une production de $1,6 \mathrm{kWh}$ d'énergie pendant les heures pleines pour $1 \mathrm{kWh}$ appelé sur le réseau pendant les heures creuses:

$$
e 1=1 / 1,6=0,625
$$

La consommation spécifique par $\mathrm{kWh}$ aux bornes de l'alternateur, calculée comme ci-dessus devient $4300 \mathrm{kcal}$ PCS $/ \mathrm{kWh}$ dont $2940 \mathrm{kcal}$ pour la chambre de combustion T.G. Ces performances correspondent au point B de la figure 3 .

\section{Accroissement des températures.}

L'augmentation de la température d'entrée turbine, permet, sans modification importante de la machine d'obtenir une puissance supérieure aux bornes de l'alternateur. Par exemple pour une température de $1000^{\circ} \mathrm{C}$ au lieu de $870^{\circ} \mathrm{C}$, on obtient $180 \mathrm{MW}$ au lieu de $160 \mathrm{MW}$, avec le même débit d'air et sans injection d'eau.

Lorsque la pression à l'admission de la turbine dépasse environ 15 bars, il est nécessaire de réaliser la détente en deux corps de machines. L'interposition d'une deuxième chambre de combustion entre ces deux corps réalise un cycle de turbine à gaz avec réchauffage intermédiaire pendant la détente. Par rapport au cycle simple, une telle disposition accroît la puissance produite, pour un débit d'air donné, ainsi que la consommation spécifique. Elle peut s'avérer intéressante pour des pressions d'accumulation supérieures à 20 bars.

\section{Accroissement des pressions.}

Les machines actuelles, particulièrement les réacteurs d'avion, ont des pressions d'admission allant jusqu'à 27 bars à l'entrée turbine (réacteur RB 211). Toutefois, elles ne sont pas adaptées à la production d'énergie par accumulation pneumatique.

Dans le cadre du matériel type adopté pour le schéma 1 , 


\section{A. CHARRIRR, I. MASCARELLO ef R. ROUDIER}

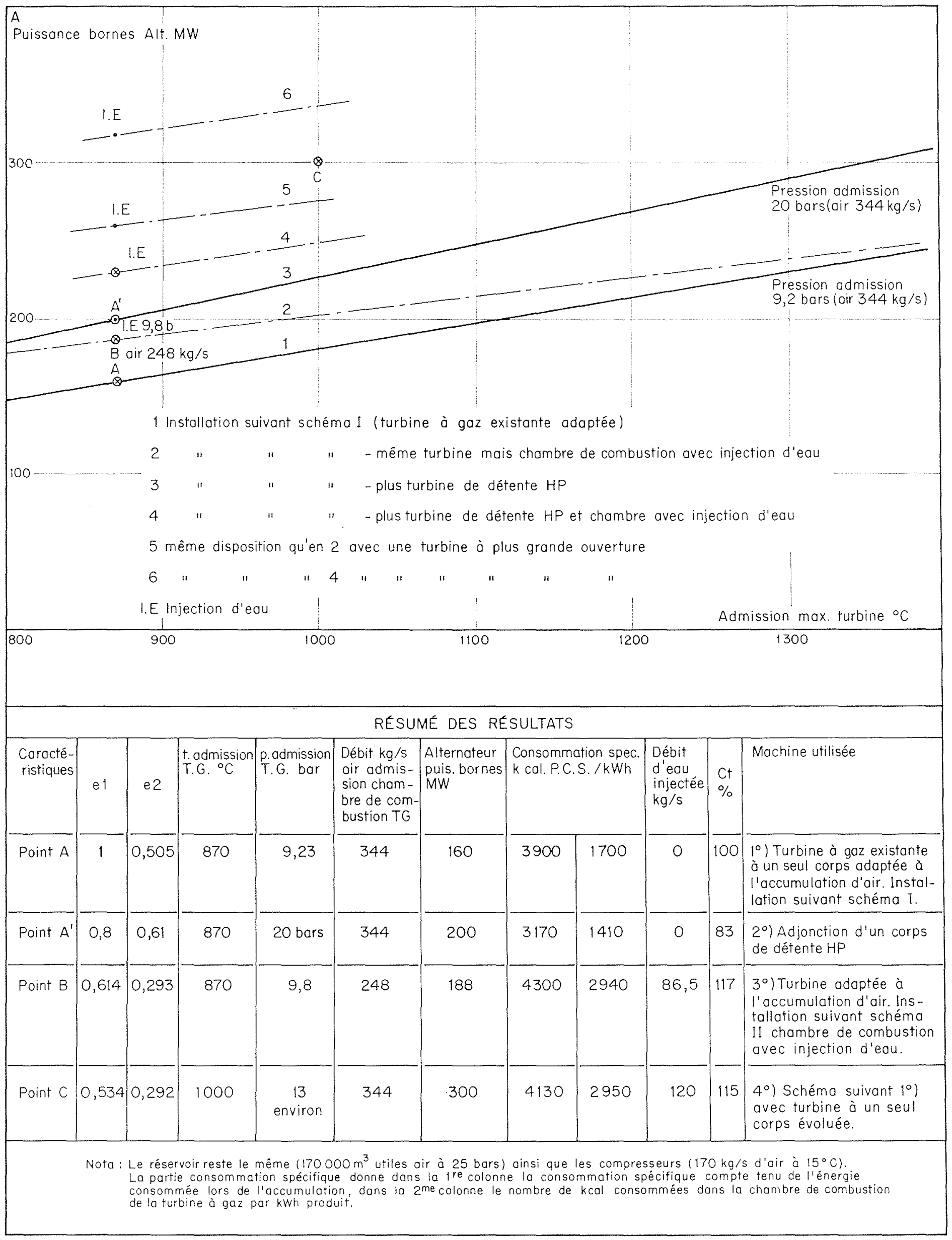

3/ Variation de la puissance aux bornes de l'alternateur en fonction de la température maxi à l'entrée de la turbine à gaz. 
il serait nécessaire d'ajouter un corps de détente HP entre 25 et 9,5 bars. La partie compression resterait inchangée.

Pour une pression d'admission de 20 bars (au lieu de 9,2) à la turbine, une température et un débit d'air identiques à ceux du schéma 1, nous obtenons les performances suivantes:

puissance aux bornes de l'alternateur $200 \mathrm{MW}$;

consommation spécifique par $\mathrm{kWh} 3170 \mathrm{kcal} / \mathrm{kWh}$ (dont 1410 pour la turbine à gaz).

Ces performances sont alors celles du point $A^{\prime}$ de la figure 3. La figure 3 résume les variantes de performances résultant soit de l'accroissement des températures ou des pressions à l'entrée de la turbine HP, soit de l'influence de l'injection d'eau.

Le point $\mathrm{C}$ correspond à une turbine à un seul corps conçue spécialement pour l'application au réservoir contenant de l'air accumulé sous 25 bars et utilisant au maximum les possibilités techniques des turbines à gaz:

- débit d'air soutiré au réservoir........ $344 \mathrm{~kg} / \mathrm{s}$;

- pression d'admission............... 13 bars;

- température d'admission maximum...... $1000^{\circ} \mathrm{C}$;

- injection d'eau correspondant à une combustion avec un excès d'air de $10 \%$.

En admettant que le constructeur accepte d'étudier une telle machine, la puissance que l'on peut espérer recueillir est de $300 \mathrm{MW}$ avec le réservoir prévu sur la figure 1.

Le tableau de la figure 3 résume les résultats que l'on pourrait attendre avec les grosses turbines à gaz actuelles plus ou moins modifiées.

\section{Estimation des coûts}

\section{Investissements.}

Compte tenu des coûts de génie civil et d'équipement du réservoir souterrain, du réservoir de surface, des coûts d'installation du matériel mécanique (y compris le transformateur élévateur de tension et les circuits de fuel), des frais d'études et d'intérêts intercalaires, une station à accumulation pneumatique conçue suivant le projet $\mathrm{A}$ a des chances d'être intéressante pour le report journalier de l'énergie électrique.

L'investissement total par $\mathrm{kW}$ installé est toutefois supérieur à celui d'une turbine à gaz simple.

Le bilan économique final dépend des aléas de construction du réservoir, de l'emplacement choisi et une caverne d'essais devrait être faite avant de passer à la réalisation d'une station hydropneumatique.

Prenons l'investissement total par $\mathrm{kW}$ pour le projet $\mathrm{A}$ égal à 100 nous obtiendrons :

- 90 pour le projet $A^{\prime}$

- 63 pour le projet $C$.

Frais proportionnels de production de l'énergie électrique $\mathrm{Ct}$.

Ils comprennent les frais de combustible. Ces frais $\mathrm{Ct}$ sont donnés sur le tableau. Si nous prenons l'indice 100 pour le projet type nous constatons que laccroissement de pression à l'admission turbine diminue ces frais (indice 83 pour le projet $A^{\prime}$ ) et que l'injection d'eau les accroit (indice 115 pour le projet $C$ ).

Pratiquement, cela signifie que la centrale à accumulation avec injection d'eau à la turbine à gaz aura un nombre d'heures d'utilisation plus faible que la centrale avec turbine à gaz classique.

\section{Conclusions}

Nous avons donné les possibilités de performances et de coût pour la partie mécanique d'une centrale à turbine à gaz installée sur un réservoir d'accumulation d'air à compensation hydraulique.

$1^{\circ}$ Aucune machine existante ne peut être utilisée dans ce but sans modification, donc sans études préalables de la part du constructeur.

$2^{\circ}$ Un premier projet A (fig. 1) très «conservateur », utilise une turbine à gaz existante, modifiée par adjonction d'un compresseur HP et probablement diverses dispositions de renforcement de paliers, modification de veine de détente, etc.

Le schéma est très simplifié : alternateur pouvant tourner en moteur - un seul accouplement débrayable à l'arrêt turbine de détente à un seul corps. La puissance obtenue est de $160 \mathrm{MW}$ pour un investissement total pris égal à 100 par $\mathrm{kW}$ produit aux bornes de l'alternateur de la turbine à gaz.

Il est possible d'obtenir $200 \mathrm{MW}$ si la température d'admission maximum passe de 870 à $1100^{\circ} \mathrm{C}$.

$3^{\circ}$ Un deuxième projet $C$, utilisant le même schéma et le même réservoir avec la même durée de production d'énergie que le projet $\mathrm{A}$, concerne une machine à développer entièrement. Il nécessite une mise en cuvre de l'injection d'eau et de toutes les possibilités technologiques permettant notamment d'avoir des températures et des pressions élevées avec un seul corps de turbine de détente.

La puissance obtenue est de $300 \mathrm{MW}$ aux bornes pour un coût d'investissement égal à $63 \%$ du coût du projet A. La réalisation suppose non seulement l'étude d'une machine nouvelle, mais aussi la mise au point d'une chambre de combustion avec injection d'eau.

Si cette technique avec injection d'eau se développe, elle n'amènera qu'une diminution de 20 à $30 \%$ du coût spécifique du matériel mais elle diminue considérablement (facteur de 1,5 à 2) le coût du réservoir par $\mathrm{kW}$ installé. Le coût global auquel on pourrait arriver devient comparable à celui des centrales à vapeur à cycle simplifié du thermique « $\mathrm{B} »$ à faible utilisation.

Les études précédentes seraient à prolonger par l'exploration de pressions d'accumulation plus élevées compatibles avec des sites bien appropriés pour réservoirs en compensation hydrauliques ou avec des réservoirs dérivés de poches salines sans compensation hydrauliques lorsque la zone d'implantation envisagée pour ce type de centrale permet géologiquement de le prévoir. 
Discussion

Président: M. P. Guyot de Villeneuve

En ouvrant la séance, M. le Président passe en revue les exposés qui figurent à son ordre du jour et conclut:

Les sujets traités par les conférenciers étant très imbriqués les uns dans les autres, je pense qu'il y aurait intérêt à reporter à l'issue des exposés, la discussion de l'ensemble des sujets abordés: caractéristiques des différents types de réservoirs et des diver cycles utilisés dans les turbines à gaz, coût d'investissement et d'exploitation, etc.

Il donne ensuite la parole à $M$. Roudier pour l'exposé de sa communication.

米

Discussion de la communication de MM. J. Mascareil.o et R. Roudier :

TECHNIQUES GÉNÉRALES

UTILISANT LE STOCKAGE DE L'AIR COMPRIME PERSPECTIVES D'AVENIR

M. le Président remercie vivement M. Roudier pour son exposé fort intéressant et sans vouloir entamc: un débat qui sera micux à sa place lors de la discussion générale, il pose au conférencier la question suivante :

Schématiquement, dans un groupe générateur électrique entraîné par turbine à gaz, celle-ci doit développer une puissance de $3 \mathrm{MW}$ pour produire $1 \mathrm{MW}$ électrique car $2 \mathrm{MW}$ sont absorbés par le compresseur alimentant la turbine à gaz.

Mais, au fur et à mesure que l'on diminue la part de puissance absorbée par le compresseur, soit en élevant la température ou la pression des gaz à l'admission, soit en injectant de l'eau au sein de ces derniers, les procédés utilisant un stockage souterrain de l'air comprimé ne deviennent-ils pas de moins en moins intéressants par rapport à une turbine à gaz fournissant la même puissance sans réservoir d'air comprimé ?

Les progrès réalisés par la turbine à gaz bénéficient au stockage d'air comprimé car, pour obtenir la meilleure rentabilité de l'ensemble de la station d'accumulation, répond M. Roubier, il est préférable d'adiopter un groupe consommant moins d'air comprimé pour une production d'énergie de pointe équivalente, le volume du réservoir est alors moins grand; or, le coût du réservoir représente une part importante du prix de l'ensemble de l'installation.

M. Mascarfllo expose son point de vue dans une longuc intervention qui peut être résumée comme suit

Les usines d'accumulation par pompage hydraulique ou par accumulation pneumatique trouveront leur application sur une grande échelle vers les années 1980, époque vers laquelle une forte partie de la production d'énergie électrique sera fournie par des centrales nucléaires de grande puissance exigeant d'importants transferts d'énergie des heures de nuit vers les heures de jour.

On aura alors besoin d'usines d'accumulation par pompage hydraulique ou pneumatique de 2000 à $3000 \mathrm{MW}$ qui devront être implantées au voisinage des centres de consommation (c'est-à-dire, non pas au fond des Alpes mais sur l'axe, Le Havre-Paris, par exemple).

Dans ces conditions, les stations d'accumulation par stockage d'air comprimé, semblent pouvoir fournir une solution satisfaisante car un réservoir d'une capacité de l'ordre d'un million de mètres cubes permet de fournir pendant six à huit heures par jour une puissance comprise entre 1000 et $2000 \mathrm{MW}$ et ce, en n'immobilisant en surface qu'une surface comparable à celle d'une usine thermique classique de même puissance.

Les études exposées par M. Roudier ont pour but de précisel ces possibilités sur la base de la technologie actuelle raisonnablement extrapolée. Cette extrapolation et encore davantage celle des coùt des turbines à gaz de l'avenir est extrêmement difficile et aléatoire. Mais les progrès vraisemblablement réalisables dans ce domaine au cours des prochaines années inclinent à l'optimisme: amélioration de la puissance maximum et du rendement des turbines à gaz par augmentation de la pression et de la température à ladmission, réduction très sensible des investissements par kilowatt de puissance installé par une construction importante et programmée des turbines à gaz, perfectionnement automatique des techniques de réalisation et d'exploitation (du fait des recherches des « pétroliers 》) et de la technologie des turbines fonctionnant à haute température (grâce aux constructeurs de propulseurs pour l'aéronantique),

M. le Président remercie MM. Roudier et Mascarello. 